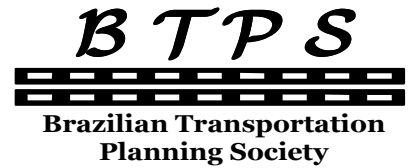

Planning Society

\author{
Journal of Transport Literature \\ Vol. 7, n. 4, pp. 147-174, Oct. 2013 \\ Research Directory
}

\section{JTL|RELIT}

www.transport-literature.org ISSN 2238-1031

\title{
Eficiência técnica: uma avaliação de aeroportos brasileiros
}

[Technical efficiency: an evaluation of Brazilian airports]

\author{
Arthur Moura Neto* \\ Universidade Candido Mendes - Brasil
}

Submitted 24 Aug 2012; received in revised form 25 Jan 2013; accepted 28 Jan 2013

\begin{abstract}
Resumo
A pesquisa apresenta uma avaliação de eficiência técnica de 23 aeroportos brasileiros, por meio do método Análise de Envoltória de Dados (DEA), tomando-se como parâmetros as relações entre a capacidade de componentes aeroportuários e o fluxo de passageiros e aeronaves. DEA tem sido utilizado em estudos para avaliação de eficiência em vários segmentos de negócio. No entanto, no que concerne a aeroportos, a relevância deste trabalho está na utilização de variáveis relativas à capacidade dinâmica de processadores de passageiros, bagagens e aeronaves - capacidade horária - para avaliação da eficiência. Adjacente aos resultados de eficiência, o estudo também identifica os componentes que comprometem o desempenho do aeroporto, bem como os acréscimos e reduções necessárias às variáveis do modelo, de modo a conduzir o aeroporto para eficiência. Os resultados obtidos indicam que $47,8 \%$ dos aeroportos operam em eficiência quando comparados com os demais aeroportos da amostra. Quanto aos componentes processadores, verifica-se que nos aeroportos identificados como ineficientes, "sala de embarque" tem o maior percentual médio em termos de necessidade de aumento de capacidade de processamento.
\end{abstract}

Palavras-Chave: análise de envoltória de dados, aeroporto, eficiência, capacidade, programação linear.

\begin{abstract}
This research presents an assessment of technical efficiency of 23 Brazilian airports, through the non-parametric technique of Data Envelopment Analysis (DEA), using as parameters the relationship between the capacity of airport components and the flow of passengers and aircraft. DEA has been used in many studies to evaluate efficiency in airports, airlines, ports, highways etc. However, with regard to airports, the relevance of this work is the use of variables related to dynamic capacity of processors passenger, baggage and aircraft - hourly capacity - for the efficiency evaluation. Adjacent to the results of efficiency, the study also identifies the components that affect the performance of the airport as well as the additions and reductions needed to model variables in order to drive to the airport efficiency. The results indicate that $47.8 \%$ of the airports operate on the frontier of efficiency when compared to other airports in the sample. As to components processors, it appears that at airports identified as inefficient, "departure lounge" has the highest average percent in terms of the need for increased processing capacity.
\end{abstract}

Key words: Data Envelopment Analysis, airport., efficiency, capacity, linear programming.

*Email: arthurmoura@infraero.gov.br.

\section{Recommended Citation}

Moura-Neto, A. (2013) Eficiência técnica: uma avaliação de aeroportos brasileiros. Journal of Transport Literature, vol. 7, n. 4, pp. 147-174.

- JTL/RELIT is a fully electronic, peer-reviewed, open access, international journal focused on emerging transport markets and published by BPTS - Brazilian Transport Planning Society. Website www.transport-literature.org. ISSN 2238-1031. 


\section{Introdução}

O objetivo deste trabalho é avaliar a eficiência técnica de uma amostra composta por 23 aeroportos brasileiros administrados pela Empresa Brasileira de Infraestrutura Aeroportuária, tomando-se como parâmetros as relações entre capacidade dinâmica de componentes aeroportuários e o fluxo de passageiros e aeronaves, fazendo uso do método não-paramétrico denominado Data Envelopment Analysis (DEA), segundo uma abordagem de retornos variáveis de escala, por considerar-se a presença de diferentes escalas nos movimentos de passageiros nos aeroportos da amostra. Com relação à representatividade da amostra, o volume de passageiros processados representou, em 2010, 92,31\% da rede INFRAERO (INFRAERO, 2011c). Para consecução do objetivo final os seguintes objetivos intermediários são estabelecidos: (i) obter, por meio do método Data Envelopment Analysis (DEA), o percentual de aeroportos da amostra que possuem índices máximos de eficiência técnica quando comparado aos demais aeroportos da amostra; (ii) identificar, dentre os aeroportos ineficientes, os componentes aeroportuários que comprometem o desempenho do aeroporto avaliado; e (iii) determinar os valores que deverão ser atribuídos aos componentes aeroportuários para que se obtenha um índice $100 \%$ de eficiência técnica. Neste sentido, os resultados obtidos nesta pesquisa podem contribuir como um instrumento adicional na gestão da infraestrutura aeroportuária.

A aceleração da economia nos últimos anos, aliada ao aumento da concorrência no setor e a consequente entrada no mercado de novos usuários do transporte aéreo, pressionaram a capacidade de processamento de aeroportos brasileiros. Este cenário conduziu a um descompasso entre o crescimento da demanda e a infraestrutura instalada.

A demanda por transporte aéreo doméstico no Brasil registrou um crescimento de $98,17 \%$ em 2010 em relação a 2005, conforme demonstrativo de passageiros-quilômetros-pagostransportados em Dados Comparativos Avançados, disponível no portal da Agência Nacional de Aviação Civil (ANAC, 2010a). Em termos de passageiros embarcados e desembarcados, os aeroportos brasileiros registraram um movimento de 155,3 milhões de passageiros em 2010. 
A planta da infraestrutura aeroportuária brasileira agrega 4.263 aeródromos e aeroportos, ocupando a segunda maior posição em nível mundial, superada apenas pela dos Estados Unidos, com 14.497 (IPEA, 2010). Da parcela de aeródromos classificada como aeroportos, 66 são administrados pela Empresa Brasileira de Infraestrutura Aeroportuária (INFRAERO), correspondendo a aproximadamente $97 \%$ do movimento do transporte aéreo regular do Brasil (INFRAERO, 2011b). No ranking dos 10 maiores aeroportos da América Latina e Caribe, em termos de passageiros embarcados e desembarcados em 2010, o aeroporto Governador André Franco Montoro (SP) ocupa a primeira posição, seguido pelo da Cidade do México, de Bogotá, de Congonhas (SP), Presidente Juscelino Kubitschek (BR), Antônio Carlos Jobim (RJ), de Cancun, Santiago do Chile, Lima e Caracas (Airport International Council apud INFRAERO (2011a). Neste contexto, faz-se importante a identificação objetiva dos fatores críticos que afetam a aviação civil, em especial quanto à compatibilidade das infraestruturas aeroportuária e aeronáutica em relação ao crescimento da demanda (IPEA, 2010). Este cenário alicerça a motivação para a seguinte formulação do problema: "A capacidade de infraestrutura aeroportuária brasileira está compatível com as demandas atuais e futuras de transporte aéreo?"

A análise quantitativa de eficiência em diferentes segmentos de negócio tem sido objeto de estudos e pesquisas. A motivação está em obter indicadores que reflitam o nível de desempenho das atividades de produção visando ações corretivas e proativas que aumentem a eficiência dos processos produtivos. Com relação aos aeroportos, a obtenção de um nível de desempenho operacional compatível com a importância do transporte aéreo conduz necessariamente à aplicação eficiente dos recursos produtivos destinados ao provimento dos serviços aeroportuários.

Os métodos de avaliação quantitativa de eficiência podem ser divididos em duas classes: paramétricos e não-paramétricos. Os métodos paramétricos ou estocásticos supõem uma relação funcional entre recursos e produtos, enquanto os não-paramétricos são baseados em programação matemática.

Conforme Lima de Souza et al (2008), o tema sobre análise de eficiência de aeroportos se tornou mais conhecido quando da publicação de resultados de pesquisa de duas instituições: o Polytechnic of Central London (PCL), hoje com a denominação de University of Westminster, e do Institut du Transport Aerien (ITA), localizado em Paris. O antigo PCL publicou, em 
1978, o estudo de Doganis et al. (1978) denominado Airport Economics in the Seventies. Nesse estudo, é realizada a análise econômica e financeira dos 22 principais aeroportos ingleses da época, além de uma abordagem sucinta da avaliação de desempenho, por meio da técnica de regressão múltipla. É considerado o primeiro trabalho formal divulgado sobre produtividade de aeroportos.

Análise do nível de eficiência de aeroportos tem sido objeto de pesquisas sob diferentes perspectivas, como financeira, operacional, de gestão e de níveis de serviço. A técnica de Análise de Envoltória de Dados predomina nas avaliações não paramétricas de eficiência. No que concerne à abordagem operacional e à aplicação do método DEA envolvendo variáveis relacionadas à capacidade de aeroportos, observa-se a utilização de componentes processadores sob a ótica de capacidade estática, como área de sala de embarque, área de pátio de aeronaves, comprimento de pista etc. Esta pesquisa, por sua vez, considera os componentes segundo seus valores de capacidade dinâmica, ou seja, a capacidade de elementos processadores de passageiros e aeronaves na unidade de tempo (hora). Considerando que a capacidade de um aeroporto depende diretamente do perfil operacional de usuários e de operadores de infraestrutura, parâmetros estes aplicados no cálculo de capacidade dinâmica (horária) de componentes processadores, este trabalho busca uma maior acuidade dos resultados da avaliação de eficiência e da identificação dos componentes aeroportuários que comprometem o desempenho do aeroporto. Informações sobre capacidade foram obtidas da base de dados da INFRAERO, processadas segundo a "Metodologia para Avaliação de Capacidade de Terminais de Passageiros", INFRAERO (2010) e por meio de consulta ao site do Centro de Gerenciamento de Navegação Aérea (CGNA, 2010).

Este artigo está dividido em 3 seções. A Seção 1 discorrre sobre aspectos gerais de infraestrutura aeroportuária, com enfase em alguns conceitos referentes à capacidade de componentes processadores. A Seção 2 explora a fundamentação teórica sobre eficiência, conforme definição de alguns autores, e o método Análise de Envoltória de Dados (DEA) e suas relações matemáticas para o cálculo de eficiência. Na sequência, a Seção 3 trata do tema central desta pesquisa. São apresentados os critérios utilizados para seleção da amostra de aeroportos e para definição das variáveis. O método DEA é aplicado para obtenção dos objetivos geral e intermédiários, bem como é realizada a análise dos resultados obtidos. Ao final, as conclusões são apresentadas. 


\section{Infraestrutura aeroportuária: aspectos operacionais}

O terminal do aeroporto é responsável por fornecer os meios e as facilidades para conduzir passageiros, bagagens e cargas oriundos do lado terra à aeronave (lado ar) e vice-versa, e planejado para permitir um fluxo adequado entre seus diversos componentes.

Segundo a International Air Transport Association, (IATA, 2011), o termo "capacidade" tem diversas variantes em termos de significado, mas, em geral, refere-se ao limite, quando alcançado ou excedido, que compromete a operação e o nível de serviço do aeroporto. A avaliação de capacidade é baseada em cinco medidas fundamentais:

\section{I) Capacidade estática}

É utilizada para descrever o potencial de armazenamento de uma área ou instalação, e está relacionada ao número de ocupantes que uma determinada área pode acomodar em um dado momento. É medida em metros quadrados por ocupante, conforme a categoria de nível de serviço ${ }^{1}$.

\section{II) Capacidade dinâmica}

Refere-se à taxa máxima de processamento de um componente por unidade de tempo. A capacidade dinâmica é comumente mensurada em termos de capacidade horária de um componente.

Conhecendo-se a capacidade estática obtém-se a capacidade dinâmica (horária) considerandose o tempo de utilização do componente processador, com base no perfil do usuário, da operação da empresa aérea, de órgãos públicos, das características dos terminais de passageiros (porte e níveis operacionais), frota em operação etc. Como exemplo, se uma sala de embarque é capaz de acomodar 50 passageiros, e esse grupo permanece na sala por 30 minutos, a capacidade é de 100 passageiros por hora.

O perfil operacional de usuários e o perfil de operadores de infraestrutura são determinantes para o cálculo da capacidade dinâmica. O primeiro está relacionado com as características

\footnotetext{
${ }^{1}$ Airport Development Reference Manual, (IATA, 1995), classifica o nível de serviço em seis diferentes categorias, identificadas como A, B, $\mathrm{C}$ e $\mathrm{D}$, conforme condições gerais de conforto, facilidade de deslocamento dentro do terminal e tempos de atendimento. O nível de serviço "C" é recomendado como o nível mínimo em um projeto, sendo definido como: bom nível de serviço, condição de fluxo estável, atrasos aceitáveis e bom nível de conforto.
} 
culturais ou econômicas dos usuários, enquanto que o segundo tem relação com o dimensionamento das equipes e com a qualidade do treinamento para execução das atividades. Exemplificando, se passageiros têm por hábito chegarem ao aeroporto com muita antecedência, (imprevisibilidade do trânsito até o aeroporto, comportamento cultural do passageiro etc) o processamento da sala de embarque pode configurar-se como "gargalo" do fluxo de passageiros em alguns períodos, devido ao maior tempo de permanência nesta área. Por outro lado, se as posições de pátio de estacionamento foram projetadas para um tempo de processamento de 40min por aeronave, mas o operador da aeronave o utiliza, em média, por 80min, a capacidade de pátio será reduzida à metade, ou seja, neste caso, o perfil do operador tem influência na redução de capacidade de um componente processador (INFRAERO, 2010). Para se conhecer o perfil do usuário e da operação é necessário realizar pesquisas periódicas para fins de atualização.

\section{III) Capacidade sustentada}

Descreve a capacidade total de um componente para acomodar, ao longo de um período de tempo, a demanda de tráfico, sob determinado nível de serviço. É a medida que combina a capacidade dinâmica e estática de componentes processadores e de áreas.

\section{IV) Capacidade máxima}

Refere-se ao fluxo máximo de tráfego que pode ser processado na unidade de tempo, mas não sustentado por longo período, mantidos os requisitos de segurança, mas sem considerar os limites de atraso e o nível de serviço.

\section{V) Capacidade declarada}

Refere-se à capacidade de um recurso ou instalação específica que limita a capacidade do aeroporto como um todo.

Com relação aos sistemas de pista e pátio, a capacidade de movimentação de aeronaves é determinada pela menor capacidade isolada entre pista e pátio.

A avaliação de capacidade do terminal de passageiros considera as dimensões físicas de áreas, os equipamentos disponíveis e as respectivas características de utilização, e o desempenho de seus componentes. Em geral, os componentes podem ser agrupados em dois tipos de 
processamento: (i) de acúmulo ou estático, em que os usuários são acumulados ao longo do tempo e liberados todos ao mesmo tempo (p.e. sala de embarque); (ii) de produção ou dinâmico, em que os usuários recebem um serviço e, logo em seguida, deixam o componente que processou o serviço (p.e. check-in). (INFRAERO, 2010).

\section{Eficiência e o método Data Envelopment Analysis}

Eficácia, produtividade e eficiência são conceitos relacionados, porém diferentes. Peña (2008) define eficácia como uma medida normativa dos alcances dos objetivos, e o nível de eficácia ou grau de consecução dos objetivos é medido por meio de produtividade média parcial, relacionando-se um produto com um de seus insumos. Porém, os indicadores de produtividade apresentam certas limitações, pois é possível que duas unidades produtivas tenham o mesmo índice de produtividade sem que uma delas esteja fazendo uso da melhor combinação de níveis de insumos e produtos.

Segundo Peña (2008), a definição de eficiência deriva do conceito de função de produção, expressa como sendo a relação entre a quantidade física de fatores de produção ou insumos e a máxima quantidade física de bens e serviços produzidos, pressupondo o método de produção mais eficiente. A Figura 1 mostra a forma geral da curva de função de produção. No trecho "OA" estão presentes retornos crescentes de escala (a produção aumenta numa proporção maior que o aumento do insumo), no trecho "AB" retornos constantes (a produção aumenta na mesma proporção que o aumento do insumo) e, a partir do ponto "B", retornos decrescentes de escala (a produção aumenta numa proporção menor que o aumento do insumo). O conjunto de alternativas de produção é formado pela área abaixo da curva de máxima produção, uma espécie de fronteira das possibilidades de produção. As unidades produtivas "A", "B e "C" estão sobre a curva e, portanto, possuem níveis de produção eficientes. No entanto, a produtividade média parcial de "A" é maior do que a de "C", pois a unidade mais produtiva é aquela cuja reta que liga o ponto à origem tem o maior coeficiente angular. Observa-se que as unidades "C" e "D" têm a mesma inclinação, logo, a mesma produtividade média parcial. Mas, “D” é ineficiente, pois para seu nível de insumo, o nível de produto seria aquele produzido por "B". Verifica-se, portanto, que níveis iguais de produtividade não necessariamente traduzem-se em mesmo nível de eficiência. Consequentemente, quando o objetivo é a avaliação do desempenho de uma unidade em 
relação a outras, no sentido do nível de otimização dos recursos utilizados, o cálculo da produtividade média por insumo não é adequado.

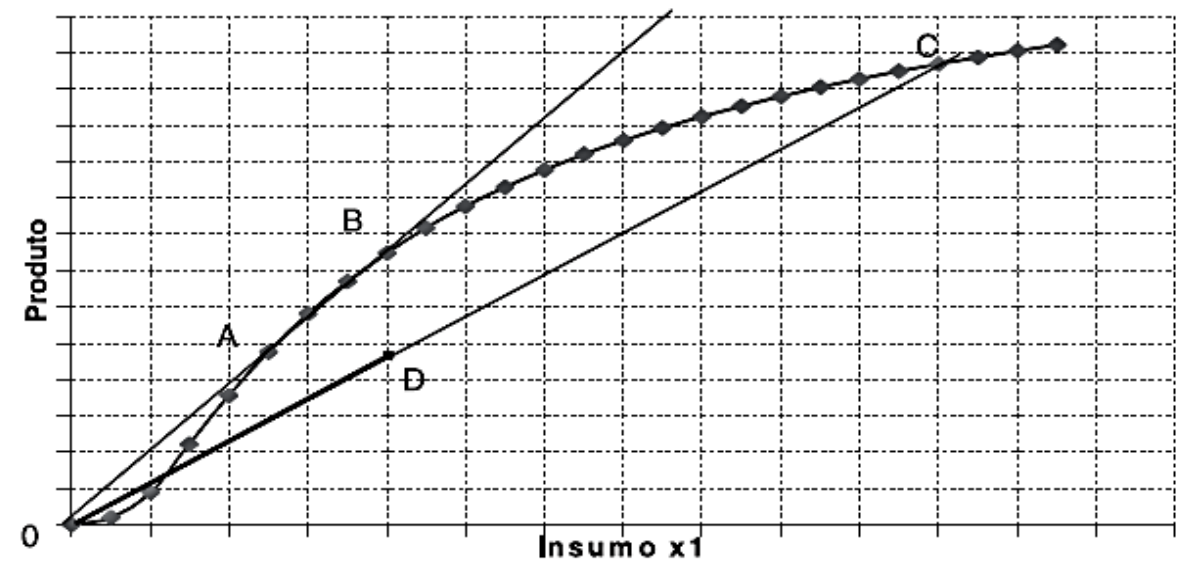

Figura 1 - Função de produção ${ }^{2}$

Conforme Toresan (1998), eficiência de um processo produtivo pode ser definida como o quociente entre a relação produto-insumo observado e a relação produto-insumo ótima. Quando este ótimo se refere ao conjunto de possibilidades de produção, diz-se que a eficiência é técnica e está associada às relações físicas entre produtos e insumos. A eficiência técnica é, então, definida como a habilidade de uma empresa em produzir o máximo de produto para um dado conjunto de insumos. Quando o ótimo considera as relações de preços entre insumos e entre produtos, tem-se a eficiência alocativa do sistema de produção. Definida a tecnologia aplicada, as combinações viáveis de produtos-insumos são estabelecidas pelo conjunto de possibilidades de produção.

Farrel (1957), em seu artigo intitulado The Measurement of Productive Efficiency, apresenta um método para medida de eficiência definida como o quociente entre um único produto (saída) e um único insumo (entrada) de um conjunto de unidades de produção. Em um plano são plotados pontos representativos das unidades de produção, cujas coordenadas são os respectivos valores de insumos e produtos. Uma linha de fronteira estabelece o conjunto de unidades eficientes, e a eficiência das demais unidades de produção é medida com base na distância de cada ponto à linha de fronteira de eficiência.

\footnotetext{
${ }^{2}$ Fonte: Peña (2008)
} 
Data Envelopment Analysis - DEA - é uma técnica baseada em programação matemática, desenvolvida por Charnes, Cooper e Rhodes (1978), destinada a medir a eficiência de unidades produtivas. DEA generaliza o método de Farrel (1957), de modo que a eficiência possa ser avaliada considerando múltiplos produtos e múltiplos insumos. As unidades produtivas são chamadas Decision Making Units (DMU), e são classificadas como eficientes ou ineficientes dentre um conjunto de DMU selecionadas para análise. As unidades eficientes estão localizadas sobre uma envoltória ou fronteira de eficiência. As ineficientes localizam-se em uma região adjacente à envoltória, e a eficiência relativa é medida com base na distância de cada DMU à linha de fronteira que contém as DMU eficientes.

O modelo representativo do método DEA pode ser orientado a maximizar a saída (orientado ao produto), ou a minimizar a entrada (orientado ao insumo). A maximização da saída é obtida mantendo-se constantes os valores de entrada (insumos) e aumentando-se os valores de saída (produtos). Para minimizar a entrada, mantém-se o nível de produto e reduzem-se os insumos. Em ambas as orientações o objetivo é aumentar a relação saída/entrada. A formulação do modelo DEA baseia-se em solução de Problema de Programação Linear (PPL) para obtenção dos índices de eficiência de cada DMU avaliada.

Existem dois modelos clássicos do método DEA: CCR, iniciais de Charnes, Cooper e Rhodes (1978), e BCC, proposto por Banker, Charnes e Cooper (1984). O CCR é direcionado para retornos constantes de escala, ou seja, variações aplicadas aos insumos (entradas) geram variações proporcionais nos produtos (saídas). O BCC considera retornos variáveis de escala a proporcionalidade entre insumos e produtos é substituída pela convexidade. Variações do CCR e BCC, como modelo Aditivo, Slacks-Based Measure of Efficiency (SBM) e Híbrido estão disponíveis na literatura.

Considerando-se as diferenças nas escalas dos movimentos de passageiros nos aeroportos da amostra, o modelo DEA utilizado neste estudo é o BCC. Adotou-se a abordagem orientada aos produtos no sentido de avaliar a necessidade de aumento de capacidade (dinâmica) de infraestrutura instalada frente à demanda de passageiros e aeronaves por componentes processadores.

A eficiência BCC orientada aos produtos é determinada por meio do PPL apresentado nas Expressões (2) a (9). O índice de eficiência da DMU avaliada $\left(\mathrm{DMU}_{\mathrm{o}}\right)$ é igual a $\frac{1}{\eta^{*}}$. $\mathrm{A} \mathrm{DMU}_{\mathrm{o}}$ 
é considerada eficiente se obtiver valor igual a 1 . Para valores $0<\frac{1}{\eta^{*}}<1$ a DMU é dita ineficiente em relação ao conjunto de DMU selecionadas na avaliação. Em um conjunto composto por $j$ DMU, os insumos e produtos de cada DMU são representados pelas matrizes $\mathrm{X}$ e $\mathrm{Y}$, respectivamente nas Equações (7) e (8). O subconjunto formado pela(s) DMUj eficiente(s) é chamado de conjunto de referência ou benchmark da $\mathrm{DMU}_{\mathrm{o}}$, definido como:

$$
E_{o}=\left\{j \mid \lambda_{j}^{*}>0\right\}(j \in\{1, \ldots, n\})
$$

Há pelo menos uma DMUj eficiente comparativamente às demais da amostra. A matriz $\lambda$ representa a contribuição das DMU eficientes para conduzir a $\mathrm{DMU}_{\mathrm{o}}$ ineficiente à fronteira da eficiência. $\boldsymbol{e}$ é um vetor linha unitário e $\boldsymbol{e} \boldsymbol{\lambda}=1$ impõe a condição de convexidade do modelo BCC (retornos variáveis de escala).

Sujeito a:

$$
\begin{gathered}
\mathrm{X} \boldsymbol{\lambda} \leq x_{o} \\
\eta y_{o}-\mathrm{Y} \boldsymbol{\lambda} \leq 0 \\
\boldsymbol{e} \boldsymbol{\lambda}=1 \\
\boldsymbol{\lambda} \geq 0 \\
\mathrm{X}=\left(\begin{array}{ccc}
x_{11} & x_{12} \cdots & x_{1 n} \\
\vdots & \ddots & \vdots \\
x_{m 1} & x_{m 2} \cdots & x_{m n}
\end{array}\right) \\
\mathrm{Y}=\left(\begin{array}{ccc}
y_{11} & y_{12} \cdots & y_{1 n} \\
\vdots & \ddots & \vdots \\
y_{s 1} & y_{s 2} \cdots & y_{s n}
\end{array}\right) \\
\boldsymbol{\lambda}=\left(\begin{array}{ccc}
\left.\lambda_{1}, \lambda_{2}, \cdots, \lambda_{n}\right) \mathrm{T}
\end{array}\right.
\end{gathered}
$$


No modelo orientado ao produto, a ineficiência é suprimida aumentando-se todas as saídas na mesma proporção. No entanto, aumentos adicionais, chamados déficits, podem, ainda, ser necessários a determinadas saídas. Analogamente, como a análise é baseada na eficiência de DMU de referência, algumas reduções nas entradas podem ser necessárias (excessos), apesar da orientação ao produto. DMU com déficit e/ou excesso posicionadas na fronteira da eficiência são ditas "fracamente eficientes".

Uma $\mathrm{DMU}_{0}$ é dita $\mathrm{BCC}$ eficiente (fortemente eficiente) se e somente se $\frac{1}{\eta^{*}}=1, \mathbf{t}^{-}=0$ (excesso) e $\mathbf{t}^{+}=0$ (déficit).

O software Frontier Analyst V. 4 (copyright 1995-2010 - Banxia Holdings Ltd) foi utilizado para o cálculo dos índices de eficiência DEA e dos demais parâmetros relacionados.

\section{Apresentação e análise dos resultados}

Os dados aplicados à pesquisa foram obtidos por meio de consulta diretamente às bases de dados da Empresa Brasileira de Infraestrutura Aeroportuária, às publicações: Estatísticas dos Aeroportos (INFRAERO, 2011c) e Projeções de Demanda por Transporte Aéreo (INFRAERO, 2011d), e ao site do Centro de Gerenciamento de Navegação Aérea (CGNA, 2010).

\subsection{Seleção dos aeroportos}

A amostra intencional selecionada para esta pesquisa é composta pelos aeroportos com movimento anual superior a 1 milhão de passageiros, o que corresponde a 23 aeroportos de um total de 66 operados pela INFRAERO. O critério para escolha foi baseado na Resolução $n^{\circ}$ 96, de 11 de maio de 2009, da Agencia Nacional de Aviação Civil que, por meio do Regulamento Brasileiro de Aviação Civil número 139 - RBAC 139 - "Certificação Operacional de Aeroportos", dispõe sobre requisitos e parâmetros de procedimentos a serem observados pelos operadores de aeródromos para a garantia da segurança das operações aeroportuárias (ANAC, 2009). Conforme o RBAC 139, “os operadores de aeródromos responsáveis por aeródromos que tenham embarcado e desembarcado mais de 1 milhão de passageiros no ano anterior ao corrente devem ser titulares de Certificado Operacional de Aeroporto". 
A Tabela 1 mostra os 23 aeroportos selecionados e os respectivos movimentos de passageiros embarcados mais desembarcados (são contabilizados os passageiros de origem mais os de conexão de origem ${ }^{3}$, bem como os passageiros de destino mais os de conexão de destino ${ }^{4}$ ), em ordem decrescente, em 2010.

\section{Tabela 1 - Aeroportos selecionados e movimento anual de passageiros ${ }^{5}$}

\begin{tabular}{|c|c|c|c|c|c|c|}
\hline Nome do Aeroporto & UF & Sigla ICAO & Doméstico & Internacional & Total & ${ }^{6} \%$ \\
\hline Gov. André Franco & SP & SBGR & 16.468 .645 & 10.380 .540 & 26.849 .185 & 18,72 \\
\hline Congonhas & SP & SBSP & 15.499 .462 & 0 & 15.499 .462 & 10,81 \\
\hline Presidente Juscelino & BR & SBBR & 14.145 .377 & 201.684 & 14.347 .061 & 10,00 \\
\hline Antônio Carlos Jobim & RJ & SBGL & 9.210 .885 & 3.127 .059 & 12.337 .944 & 8,60 \\
\hline Santos Dumont & RJ & SBRJ & 7.822 .848 & 0 & 7.822 .848 & 5,45 \\
\hline Dois de Julho & BA & SBSV & 7.350 .486 & 345.821 & 7.696 .307 & 5,37 \\
\hline Tancredo Neves & MG & SBCF & 6.959 .577 & 301.487 & 7.261 .064 & 5,06 \\
\hline Salgado Filho & RG & SBPA & 6.227 .568 & 448.648 & 6.676 .216 & 4,66 \\
\hline Guararapes & $\mathrm{PE}$ & SBRF & 5.750 .799 & 208.183 & 5.958 .982 & 4,16 \\
\hline Afonso Pena & PR & SBCT & 5.671 .273 & 103.342 & 5.774 .615 & 4,03 \\
\hline Viracopos & SP & SBKP & 5.387 .577 & 42.489 & 5.430 .066 & 3,79 \\
\hline Pinto Martins & $\mathrm{CE}$ & SBFZ & 4.843 .258 & 229.463 & 5.072 .721 & 3,54 \\
\hline Eduardo Gomes & $\mathrm{AM}$ & SBEG & 2.528 .999 & 159.624 & 2.688 .623 & 1,87 \\
\hline Hercílio Luz & $\mathrm{SC}$ & SBFL & 2.478 .905 & 193.345 & 2.672 .250 & 1,86 \\
\hline Eurico de A. Salles & ES & SBVT & 2.644 .729 & 0 & 2.644 .729 & 1,84 \\
\hline Val de Cães & PA & SBBE & 2.562 .783 & 42.684 & 2.605 .467 & 1,82 \\
\hline Augusto Severo & $\mathrm{RN}$ & SBNT & 2.297 .778 & 118.055 & 2.415 .833 & 1,68 \\
\hline Santa Genoveva & GO & SBGO & 2.346 .564 & 2.084 & 2.348 .648 & 1,64 \\
\hline Marechal Rondon & MT & SBCY & 2.132 .983 & 1.284 & 2.134 .267 & 1,49 \\
\hline Zumbi dos Palmares & $\mathrm{AL}$ & SBMO & 1.411 .561 & 20.220 & 1.431 .781 & 1,00 \\
\hline M. Hugo Machado & MA & SBSL & 1.378 .061 & 1.085 & 1.379 .146 & 0,96 \\
\hline Campo Grande & MS & SBCG & 1.193 .343 & 15.422 & 1.208 .765 & 0,84 \\
\hline Cataratas & PR & SBFI & 1.138 .572 & 17.043 & 1.155 .615 & 0,81 \\
\hline
\end{tabular}

\footnotetext{
${ }^{3}$ Passageiros de conexão de origem são aqueles que desembarcam e reembarcam sem reprocessamento

${ }^{4}$ Passageiros de conexão de destino são aqueles que reembarcam depois de processados na conexão

${ }^{5}$ Fonte: (INFRAERO, 2011c) - adaptado pelo autor

${ }^{6}$ Participação percentual do aeroporto em relação à amostra
} 


\subsection{Definição das variáveis}

Uma questão crítica do método Análise de Envoltória de Dados diz respeito à relação entre quantidade de DMU (neste estudo, aeroportos) e o número de variáveis. À medida que aumenta o número de variáveis em relação ao número de unidades produtivas, menor é a capacidade de ordenação pelas eficiências, pois muitas unidades tendem a se posicionar sobre a fronteira. Segundo Cooper et al (2007), o número de DMU (n) deve se relacionar com o número de insumos (m) e produtos (s) conforme a Expressão (10).

$$
\mathrm{n} \geq \max \{\mathrm{mx} \mathrm{s} ; 3(\mathrm{~m}+\mathrm{s})\}
$$

A Tabela 2 mostra as variáveis consideradas previamente à seleção para atendimento à Expressão (10). A coluna Entrada (E) ou Saída (S) indica se as variáveis são insumos ou produtos, respectivamente.

Com relação ao movimento de passageiros na hora pico, variável PAX_HP, a definição de Hora Pico adotada pela INFRAERO tem como referência o artigo de Wang, P.T. e Pitfield, D.E (1999). As demandas horárias de um aeroporto registradas ao longo de um ano são descritas em percentuais do movimento anual, em ordem decrescente. Esses percentuais são somados, até que se obtenha uma participação acumulada de 3,5\% do movimento anual, que representa o atendimento de $96,5 \%$ da demanda.

Neste sentido e sob a ótica das variáveis mais representativas para aplicação da DEA, métodos estatísticos e técnicas multicritério são comumente utilizados para seleção das variáveis. Neste trabalho adotou-se a Análise Fatorial (AF) como instrumento de suporte à composição das variáveis aplicadas à DEA. Hair et al (apud Pires de Almeida, 2009) entende que a principal característica que conduz à utilização da Análise Fatorial seja a necessidade de resumir os dados e reduzir o número de variáveis. As variáveis são agrupadas em função das suas altas correlações e os agrupamentos resultantes tornam-se novas variáveis em número menor e mais abrangente de fenômenos mais gerais. Essas novas variáveis são chamadas fatores. Para extração dos fatores, a Análise de Componentes Principais é o método mais conhecido, o qual consiste em transformar um conjunto de variáveis iniciais, correlacionadas entre si, em outro conjunto de variáveis não correlacionadas, denominadas componentes principais, que são combinações lineares do conjunto inicial. A associação específica entre 
fatores e variáveis originais é determinada pela carga fatorial. A carga fatorial consiste em um coeficiente (um número decimal, positivo ou negativo, geralmente menor do que a unidade) que expressa o quanto uma variável observada está carregada em um fator. Pode-se dizer que quanto maior é a carga em cima de um fator, mais a variável se identifica com o fator que a representa (Vicini, 2005).

Tabela 2 - Variáveis inicialmente consideradas ${ }^{7}$

\begin{tabular}{|l|l|l|c|}
\hline Nome da variável & Un. & Descrição & $\begin{array}{c}\text { Entrada (E) } \\
\text { ou Saída (S) }\end{array}$ \\
\hline PAX_REG_ANO & pax/ano & Movimento anual de passageiros em voo regular & (E) \\
\hline PAX_NREG_ANO & pax/ano & Movimento anual de passageiros em voo não regular & (E) \\
\hline PAX_ANO & pax/ano & Movimento anual total de passageiros & (E) \\
\hline PAX_HP & pax/h & Movimento de passageiros na hora pico & (E) \\
\hline ARNV_REG_ANO & mov/ano & Movimento anual de aeronave em voo regular & (E) \\
\hline ARNV_NREG_ANO & mov/ano & Movimento anual de aeronave em voo não regular & (E) \\
\hline ARNV_ANO & mov/ano & Movimento anual total de aeronave & (E) \\
\hline ARNV_HP & mov/h & Movimento de aeronave na hora pico & (E) \\
\hline PATIO_HP & mov/h & Concentração de aeronave no pátio na hora pico & (E) \\
\hline PISTA & mov/h & Capacidade de pista de pouso e decolagem & (S) \\
\hline CHECK_IN & pax/h & Capacidade dos balcões de check-in & (S) \\
\hline AREA_CHECK-IN & pax/h & Capacidade de área de fila do check-in & (S) \\
\hline SALA_EMB & pax/h & Capacidade de sala de embarque & (S) \\
\hline SALA_DES & pax/h & Capacidade de sala de desembarque & (S) \\
\hline REST_BAG & pax/h & Capacidade de esteira de restituição de bagagem & \\
\hline
\end{tabular}

As relações entre as variáveis podem ser verificadas por meio da matriz de coeficiente de correlação linear, de modo que seja medida a associação entre cada par de variáveis. A técnica de AF permitirá concluir se é possível explicar esse padrão de correlações, mediante um menor número de variáveis. Os autovalores representam o número total de componentes que melhor explicam o conjunto de variáveis originais. O autovalor representa a proporção da variância total explicada por cada fator, ou seja, avalia a contribuição do fator ao modelo

\footnotetext{
${ }^{7}$ Fonte: autor

${ }^{8}$ Tráfego regido pelo HOTRAN (possui frequência e horários regulares, conhecidos como voos de "carreira").

${ }^{9}$ Refere-se aos voos charter, aviação executiva, agrícola etc.
} 
construído pela análise fatorial. Os autovetores são os coeficientes de ponderação associados às variáveis originais na formação das combinações lineares (Vicini, 2005).

As operações matemáticas envolvidas na Análise Fatorial são viabilizadas por meio do uso de softwares de análise especiais. Neste estudo, foi utilizado o software Statistica V.10 para extração e análise dos fatores que serviram de suporte à seleção das variáveis aplicadas à pesquisa.

A redução do número de variáveis nesta pesquisa visa atender duas fundamentações. A primeira, refere-se à relação entre a quantidade de aeroportos e o número de variáveis, descrita na Expressão (10). A segunda, diz respeito à representatividade das variáveis, na qual se busca identificar o relacionamento entre o conjunto de dados visando à utilização de um número menor de fatores (variáveis) que melhor representem as variáveis originais.

Uma restrição do método DEA diz respeito à transformação dos dados de entrada e/ou saída, ou seja, a solução do PPL é afetada caso os valores das variáveis passem por um processo de transformação, em geral para conversão de valores negativos em positivos. Alguns modelos DEA são ditos translation invariant: transformações aplicadas nos valores das entradas e/ou saídas não alteram os resultados dos cálculos de eficiência. O modelo CCR não compartilha desta propriedade; no BCC a propriedade é limitada, no sentido de que na orientação ao insumo a transformação somente é invariante para os produtos, e na orientação ao produto a transformação só pode ser aplicada aos insumos (Cooper, Seiford e Tone, 2007).

Em face às observações supracitadas, e considerando que na extração dos fatores pelo método dos componentes principais as novas variáveis são uma combinação linear dos dados originais, a Análise Fatorial é utilizada, nesta pesquisa, para avaliação e seleção dos dados mais representativos, sem, no entanto, promover a substituição de variáveis originais por componentes principais, devido às transformações a que os dados são submetidos.

A escolha do número de autovalores e, consequentemente, da quantidade de fatores pode obedecer a diferentes critérios. Um deles inclui as componentes que sintetizam uma variância acumulada em torno de 70\% (Vicini 2005). Pode-se, também, fazer a seleção incluindo-se somente aquelas componentes cujos valores próprios são superiores a 1 (Kaiser apud Vicini, 2005). Assim, definiu-se que os autovalores considerados seriam aqueles superiores a 0,1 . 
A análise de fatores aplicada às variáveis de entrada é apresentada na Tabela 3.

Tabela 3 - Autovalores (entrada) ${ }^{10}$

\begin{tabular}{|c|r|r|r|r|}
\hline Fatores & Autovalor & Variância (\%) & $\begin{array}{r}\text { Autovalor } \\
\text { acumulado }\end{array}$ & $\begin{array}{r}\text { Variância } \\
\text { acumulada (\%) }\end{array}$ \\
\hline 1 & 7,755345 & 86,17050 & 7,755345 & 86,17050 \\
\hline 2 & 0,766326 & 8,51473 & 8,521671 & 94,68523 \\
\hline 3 & 0,423961 & 4,71068 & 8,945632 & 99,39591 \\
\hline
\end{tabular}

Os resultados apontaram para três fatores, totalizando cerca de $99 \%$ da variância total dos dados. O próximo passo seria a obtenção dos autovetores, que são os coeficientes de ponderação das variáveis originais para geração dos valores dos novos fatores (variáveis). Como foram mantidas as variáveis originais, devido às restrições do método DEA descritas anteriormente, dispensou-se a demonstração deste resultado.

A contribuição de cada variável (original) nos três fatores é visualizada por meio da carga fatorial, como mostra a Tabela 4. Observa-se que, com exceção de PAX_NREG_ANO e ARNV_NREG_ANO, a carga fatorial das variáveis no Fator 1 é bastante expressiva, em especial PAX_HP e ARNV_REG_ANO,com valores praticamente iguais.

Tabela 4 - Carga fatorial (entrada) ${ }^{11}$

\begin{tabular}{|l|r|r|r|}
\hline Variável & \multicolumn{1}{|l|}{ Fator 1 } & \multicolumn{1}{l|}{ Fator 2 } & \multicolumn{1}{l|}{ Fator 3 } \\
\hline PAX_REG_ANO & 0,851931 & 0,234566 & 0,464904 \\
\hline PAX_NREG_ANO & 0,374496 & 0,142227 & 0,915437 \\
\hline PAX_ANO & 0,845097 & 0,233604 & 0,477891 \\
\hline PAX_HP & 0,895456 & 0,249795 & 0,350656 \\
\hline ARNV_REG_ANO & 0,895240 & 0,282342 & 0,338945 \\
\hline ARNV_NREG_ANO & 0,314794 & 0,939100 & 0,130053 \\
\hline ARNV_ANO & 0,841618 & 0,431157 & 0,320788 \\
\hline ARNV_HP & 0,806092 & 0,515571 & 0,271853 \\
\hline PATIO_HP & 0,863500 & 0,450905 & 0,190544 \\
\hline
\end{tabular}

\footnotetext{
${ }^{10}$ Fonte: autor

${ }^{11}$ Fonte: autor
} 
Optou-se por utilizar a variável PAX_HP por considerar que:

- É a variável que indica o período de maior solicitação dos componentes de infraestrutura aeroportuária;

- O valor de hora-pico considerado no presente estudo inclui o movimento de passageiros em voos regulares e não regulares;

- Apresenta comportamento diferenciado em função do perfil de demanda (negócios, turismo/lazer) do aeroporto. Aeroportos com menor densidade de tráfego tendem a apresentar valores de PAX_HP concentrados, contrapondo-se com "vales" durante o dia, enquanto que os de maior densidade se caracterizam por uma distribuição quase uniforme da demanda; e

- Das seis variáveis de saída, cinco referem-se à capacidade de processadores do terminal de passageiros.

Com relação ao Fator 2, a carga fatorial da variável ARNV_NREG_ANO é predominante. Justifica-se a inclusão desta variável por apresentar características peculiares, como:

- Este tráfego, em geral, não utiliza as facilidades do terminal de passageiros, mas tem forte influência na capacidade do sistema de pista e do pátio de aeronaves;

- Apesar de registrar um pequeno volume de passageiros, quando comparado ao tráfego regular, tem alta capilaridade (opera em mais de 4000 aeródromos, contra pouco mais de 130 operados pela aviação regular); e

- O Brasil conta atualmente com a $2^{\mathrm{a}}$ maior frota mundial de aviação executiva e este segmento é considerado como termômetro do nível de atividade econômica de um país.

Quanto ao Fator 3, PAX_NREG_ANO é a que possui maior peso. No entanto, por representar uma pequena parcela do movimento total de passageiros e ser considerada no valor de hora pico, não foi incluída.

Os fatores com os respectivos autovalores obtidos na análise das variáveis de saída são mostrados na Tabela 5 . 
Tabela 5 - Autovalores (saída) ${ }^{12}$

\begin{tabular}{|c|r|r|r|r|}
\hline Fatores & Autovalor & Variância (\%) & $\begin{array}{r}\text { Autovalor } \\
\text { acumulado }\end{array}$ & $\begin{array}{r}\text { Variância } \\
\text { acumulada (\%) }\end{array}$ \\
\hline 1 & 4,192841 & 69,88068 & 4,192841 & 69,8807 \\
\hline 2 & 0,948646 & 15,81076 & 5,141487 & 85,6914 \\
\hline 3 & 0,492578 & 8,20964 & 5,634065 & 93,9011 \\
\hline 4 & 0,277710 & 4,62849 & 5,911774 & 98,5296 \\
\hline
\end{tabular}

Para autovalores maiores que 0,1 foram extraídos quatro fatores com variância total acumulada de $98 \%$. A carga fatorial em cada um dos fatores é mostrada na Tabela 6.

Tabela 6 - Carga fatorial (saída $)^{13}$

\begin{tabular}{|l|r|r|r|r|}
\hline Variável & \multicolumn{1}{|l|}{ Fator 1 } & \multicolumn{1}{l|}{ Fator 2 } & \multicolumn{1}{l|}{ Fator 3 } & \multicolumn{1}{l|}{ Fator 4 } \\
\hline PISTA & 0,119278 & 0,940053 & 0,109858 & 0,299908 \\
\hline CHECK_IN & 0,311437 & 0,311913 & 0,356279 & 0,811560 \\
\hline AREA_CHECK-IN & 0,441214 & 0,358370 & 0,059643 & 0,809573 \\
\hline SALA_EMB & 0,348754 & 0,109017 & 0,914622 & 0,172998 \\
\hline SALA_DES & 0,804912 & 0,172994 & 0,425596 & 0,315917 \\
\hline REST_BAG & 0,857997 & 0,105359 & 0,300308 & 0,380019 \\
\hline
\end{tabular}

SALA_DES e REST_BAG dominam as cargas fatoriais no Fator 1. O mesmo efeito é observado quanto à CHECK-IN e à AREA_CHECK-IN no Fator 4. Visando anular redundância na aplicação da DEA, as variáveis REST_BAG e CHECK-IN foram selecionadas por apresentarem os maiores pesos nos respectivos fatores.

As variáveis PISTA e SALA_EMB têm alto destaque nos Fatores 2 e 3, respectivamente, sendo, portanto, selecionadas.

As variáveis que compõem o estudo são, portanto:

- PAX_HP (entrada)

- ARNV_NREG_ANO (entrada)

\footnotetext{
${ }^{12}$ Fonte: autor

${ }^{13}$ Fonte: autor
} 
- REST_BAG (saída)

- CHECK-IN (saída)

- SALA_EMB (saída)

- PISTA (saída)

Quanto à Expressão (10), é satisfeita a relação entre o número de DMU (n), de insumos (m) e de produtos (s).

A Tabela 7 apresenta os índices de eficiência obtidos por meio do método DEA para os 23 aeroportos.

Tabela 7 - Índice de eficiência $(\mathrm{BCC}-\mathrm{O})^{14}$

\begin{tabular}{|l|c|c|c|}
\hline \multicolumn{1}{|c|}{ Nome do Aeroporto } & UF & Sigla ICAO & Eficiência $\left.\boldsymbol{( \theta}^{*}\right)$ \\
\hline Val de Cães & PA & SBBE & 1,000 \\
\hline Tancredo Neves & MG & SBCF & 1,000 \\
\hline Augusto Severo & RN & SBNT & 1,000 \\
\hline Congonhas & SP & SBSP & 1,000 \\
\hline Presidente Juscelino Kubitschek & BR & SBBR & 1,000 \\
\hline Campo Grande & MS & SBCG & 1,000 \\
\hline Cataratas & PR & SBFI & 1,000 \\
\hline Antônio Carlos Jobim & RJ & SBGL & 1,000 \\
\hline Gov. André Franco Montoro & SP & SBGR & 1,000 \\
\hline Guararapes & PE & SBRF & 1,000 \\
\hline Zumbi dos Palmares & AL & SBMO & 1,000 \\
\hline Viracopos & SP & SBKP & 0,957 \\
\hline Dois de Julho & BA & SBSV & 0,851 \\
\hline Afonso Pena & PR & SBCT & 0,832 \\
\hline Hercílio Luz & SC & SBFL & 0,802 \\
\hline Santos Dumont & RJ & SBRJ & 0,799 \\
\hline Eduardo Gomes & AM & SBEG & 0,788 \\
\hline Marechal Rondon & MT & SBCY & 0,783 \\
\hline Santa Genoveva & GO & SBGO & 0,768 \\
\hline Mar. Hugo da C. Machado & MA & SBSL & 0,754 \\
\hline Pinto Martins & CE & SBFZ & 0,704 \\
\hline Eurico de A. Salles & ES & SBVT & 0,682 \\
\hline Salgado Filho & RG & SBPA & 0,620 \\
\hline
\end{tabular}

\footnotetext{
${ }^{14}$ Fonte: autor
} 
Observa-se na Tabela 7 que dos vinte e três aeroportos da amostra, onze obtiveram índice $\theta^{*}=$ 1, ou seja, $47,8 \%$ do total são considerados relativamente eficientes. A eficiência média é de 0,88 , com desvio padrão igual a 0,12 .

O Aeroporto Internacional Salgado Filho obteve o menor índice $\left(\theta^{*}=0,62\right)$, cabendo algumas observações. SBPA é o principal aeroporto da região sul do país, com movimento superior a 6,5 milhões de passageiros em 2010. É a porta de entrada/saída do/para o MERCOSUL e o centro de operações (hub) da GOL Linhas Aéreas para voos com destino ao Cone Sul, concentrando conexões para Buenos Aires, Santiago, Rosário, Córdoba e Montevidéu. Como Porto Alegre não possui aeroportos auxiliares metropolitanos, SBPA concentra todo o tráfego de aeronaves de pequeno e grande porte.

Uma análise quanto à distribuição do volume de passageiros ao longo do dia da hora-pico indica que $67 \%$ dos aeroportos ineficientes possuem densidade relativamente uniforme SBKP, SBSV, SBCT, SBFL, SBRJ, SBFZ, SBVT e SBPA. Como não são aeroportos com perfil de tráfego com "vales" acentuados, e por processarem um volume anual de passageiros na faixa de 2,6 milhões a 7,8 milhões, são bastante sensíveis a atrasos.

A Figura 2 ilustra como estão distribuídas as eficiências; no eixo horizontal estão as faixas dos índices de eficiência e no eixo vertical o número de aeroportos pertencentes a cada faixa.

A faixa entre $71 \%$ e $80 \%$ concentra as maiores ineficiências, com seis aeroportos: SBFL, SBRJ, SBEG, SBCY, SBGO e SBSL. O Aeroporto Mar. Hugo da Cunha Machado (SBSL) está no limite inferior desta faixa de eficiência, $\operatorname{com} \theta^{*}=0,75$. Ressalta-se que SBSL registrou em 2010 um aumento de cerca de $40 \%$ no movimento de passageiros quando comparado com 2009 e de $22 \%$ no movimento de aeronaves no mesmo período. 
Figura 2 - Distribuição das eficiências ${ }^{15}$

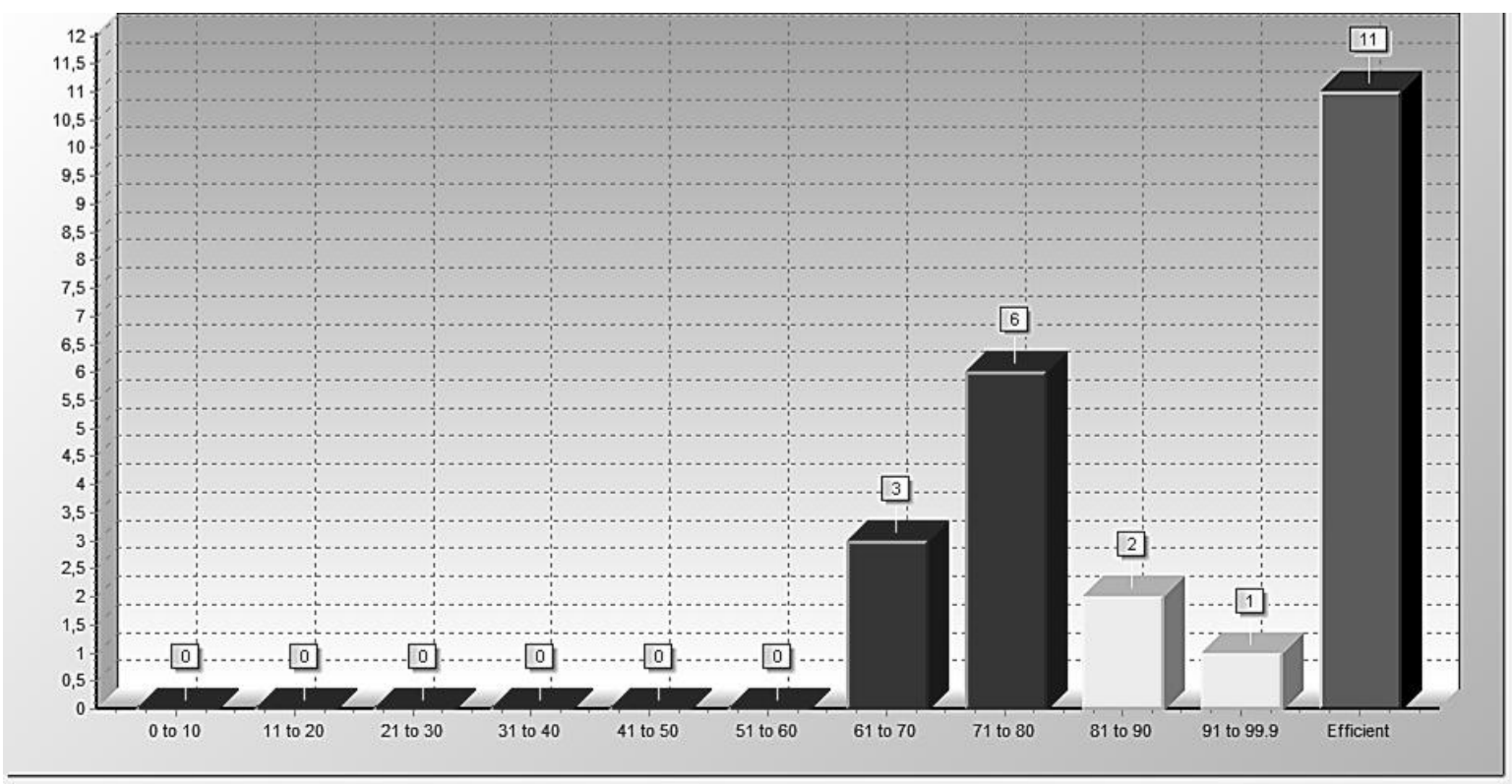

Em "Demanda na Hora-Pico dos Aeroportos da Rede INFRAERO" (ANAC, 2007) é apresentada uma metodologia para obtenção do índice de concentração de demanda na hora pico, definido como a relação entre o quantitativo de passageiros na hora-pico e o movimento anual. O modelo identifica grupos de faixas de movimento anual de passageiros com clusters de aeroportos com comportamentos semelhantes quanto à concentração de demanda na horapico. Com base nessas faixas, sugere-se uma classificação quanto ao porte do aeroporto, baseada no volume de passageiros processados anualmente: pequeno (até 999,9 mil), médio (1 milhão até 2,9 milhões), grande (3 milhões até 7,9 milhões) e extragrande (acima de 8 milhões). Com base nesta metodologia e reportando-se à Tabela 1, verifica-se que todos os aeroportos categorizados como extragrande são eficientes: SBGR, SBSP, SBBR e SBGL. Esses aeroportos processaram, em 2010, 48,13\% do total de passageiros da amostra e 44,43\% da rede INFRAERO. Constata-se, também, que, com exceção dos aeroportos SBCF e SBRF, identificados como eficientes, os aeroportos pertencentes à faixa grande porte obtiveram índices $\theta^{*}<1$ : SBKP, SBSV, SBCT, SBRJ, SBFZ e SBPA.

A Tabela 8 mostra os percentuais de ampliação de capacidade de cada componente processador para posicionamento do aeroporto sobre a fronteira da eficiência. Observa-se que

${ }^{15}$ Fonte: autor 
a eficiência $\theta^{*}=1$ para o aeroporto SBPA é alcançada ampliando-se as capacidades de pista, balcão de check-in e sala de embarque em $61 \%$, e em $84 \%$ a capacidade de esteira de restituição de bagagem. Por outro lado, ajustes seriam necessários no movimento anual de aeronaves não regulares, com redução de $23 \%$. Dois aspectos devem ser considerados com relação ao SBPA: (i) o aeroporto possui dois Terminais de Passageiros e um Terminal de Cargas (TECA), porém, o Terminal 2 esteve por muito tempo desativado, sendo reaberto em dezembro de 2010 para voos domésticos, disponibilizando, assim, novos recursos para processamento de passageiros e bagagens. (ii) como consequência do primeiro, os dados de capacidade do segundo Terminal não foram contabilizados no cálculo de eficiência.

Os aeroportos SBGO, SBCY e SBFL mostraram necessidades de ajustes superiores a $100 \%$ na capacidade das salas de embarque, sinalizando intervenções para ampliação espacial do Terminal de Passageiros.

Tabela 8 - Ajustes de capacidade ${ }^{16}$

\begin{tabular}{|l|c|c|c|c|c|c|}
\hline $\begin{array}{l}\text { NOME DO } \\
\text { AEROPORTO }\end{array}$ & $\begin{array}{l}\text { Sigla } \\
\text { ICAO }\end{array}$ & PISTA & CHECK-IN & SALA_EMB & REST_BAG & \multicolumn{2}{l|}{$\begin{array}{l}\text { ARNV_NREG_ } \\
\text { ANO }\end{array}$} \\
\hline Viracopos & SBKP & $+5 \%$ & $+42 \%$ & $+4 \%$ & $+4 \%$ & $0 \%$ \\
\hline Dois de Julho & SBSV & $+17 \%$ & $+75 \%$ & $+17 \%$ & $+17 \%$ & $-52 \%$ \\
\hline Afonso Pena & SBCT & $+20 \%$ & $+120 \%$ & $+20 \%$ & $+20 \%$ & $-13 \%$ \\
\hline Hercílio Luz & SBFL & $+24 \%$ & $+24 \%$ & $+112 \%$ & $+24 \%$ & $-28 \%$ \\
\hline Santos Dumont & SBRJ & $+25 \%$ & $+25 \%$ & $+25 \%$ & $+59 \%$ & $-22 \%$ \\
\hline Eduardo Gomes & SBEG & $+29 \%$ & $+26 \%$ & $+26 \%$ & $+32 \%$ & $-40 \%$ \\
\hline $\begin{array}{l}\text { Marechal } \\
\text { Rondon }\end{array}$ & SBCY & $+27 \%$ & $+27 \%$ & $+120 \%$ & $+27 \%$ & $-53 \%$ \\
\hline Santa Genoveva & SBGO & $+30 \%$ & $+30 \%$ & $+122 \%$ & $+30 \%$ & $-71 \%$ \\
\hline $\begin{array}{l}\text { Mar. Hugo da } \\
\text { C. Machado }\end{array}$ & SBSL & $+32 \%$ & $+32 \%$ & $+32 \%$ & $+58 \%$ & $0 \%$ \\
\hline Pinto Martins & SBFZ & $+42 \%$ & $+65 \%$ & $+42 \%$ & $+42 \%$ & $-25 \%$ \\
\hline $\begin{array}{l}\text { Eurico de A. } \\
\text { Salles }\end{array}$ & SBVT & $+46 \%$ & $+46 \%$ & $+74 \%$ & $+114 \%$ & $-59 \%$ \\
\hline Salgado Filho & SBPA & $+61 \%$ & $+61 \%$ & $+61 \%$ & $+84 \%$ & $-23 \%$ \\
\hline
\end{tabular}

\footnotetext{
${ }^{16}$ Fonte: autor
} 
A Tabela 9 sumariza os valores percentuais médios de ineficiências das variáveis utilizadas no modelo. Verifica-se que a componente sala de embarque tem o maior percentual médio em termos de necessidade de aumento de capacidade $(26,32 \%)$.

Tabela 9 - Percentuais médios de ineficiência das variáveis ${ }^{17}$

\begin{tabular}{|l|c|}
\hline \multicolumn{1}{|c|}{ VARIÁ VEIS } & $\begin{array}{c}\text { PERCENTUAIS } \\
\text { MÉDIOS (\%) }\end{array}$ \\
\hline PAX_HP (mov. pax na HP) & 0 \\
\hline ARNV_NREG_ANO (mov. anual não reg.) & $-15,6$ \\
\hline PISTA (c. de pista de P/D - mov/h) & 14,47 \\
\hline CHECK-IN (c. balcão de check-in - pax/h) & 23,06 \\
\hline SALA_EMB (c. sala de embarque - pax/h) & 26,32 \\
\hline REST_BAG (c. esteira de bagagem - pax/h) & 20,55 \\
\hline
\end{tabular}

Para que uma DMU ineficiente alcance $\theta^{*}=1$ é preciso que todas as entradas (insumos) e saídas (produtos) sejam ajustadas. Esta é uma condição fundamental da metodologia DEA. No contexto deste estudo, a ampliação parcial da capacidade de componentes ineficientes não conduz $\theta^{*}$ para o valor máximo. A interpretação desta condição na infraestrutura aeroportuária torna-se clara quando, em determinado fluxo operacional, se considera a interdependência dos componentes e elementos processadores de passageiros e bens.

Para exemplificar, no caso de o check-in e a área de embarque de um Terminal de Passageiros necessitarem de ampliações, um aumento da capacidade somente em relação aos balcões não seria suficiente para melhorias, uma vez que as limitações da sala de embarque continuariam a configurar ineficiência no processamento de embarque de passageiros como um todo.

A relatividade do conceito de eficiência é outra fundamentação importante da DEA. Para ilustrar, toma-se o Aeroporto Internacional Eduardo Gomes (SBEG): as ampliações das capacidades dos balcões de check-in e da sala de embarque em $26 \%$, e da esteira de restituição de bagagem em $32 \%$, são requeridas quando comparadas às do Aeroporto Internacional Val de Cães (SBBE); deste modo, dada as mesmas entradas e saídas da amostra e seus respectivos valores, SBBE é benchmark para SBEG.

A fim de verificar se aeroportos considerados relativamente eficientes no modelo BCC interceptam a fronteira CCR, os vinte e três aeroportos da amostra foram aplicados ao modelo

\footnotetext{
${ }^{17}$ Fonte: autor
} 
CCR. Os resultados mostraram que dos onze eficientes-BCC, nove estão operando na fronteira CCR: SBBE, SBCF, SBNT, SBSP, SBCG, SBFI, SBGL, SBRF e SBMO. Portanto, pressupondo-se que operam com retornos constante de escala, um aumento no volume de passageiros na hora-pico implica aumento de capacidade de componentes processadores na mesma proporção. SBGR e SBBR, eficientes no BCC, apresentam eficiência relativa de $86,40 \%$ e $55,30 \%$ no CCR, respectivamente.

Uma análise preliminar sobre os aeroportos que estão na fronteira da eficiência-BCC conduz à interpretação de que operam no limite de suas capacidades, pois um aumento na demanda de passageiros implica em um deslocamento para fora da fronteira, isto é, em uma saturação de capacidade. No entanto, há de se considerar o fator de escala, tanto em termos da natureza (crescente ou decrescente) quanto da magnitude. Em caso de operação com retornos decrescentes de escala, um aumento na demanda requer um aumento de capacidade à razão menor. Supondo-se que em resposta a um aumento da demanda à determinada taxa, o deslocamento da capacidade necessário ao reposicionamento do aeroporto sobre a fronteira seja à taxa substancialmente menor, características inerentes à própria operação do aeroporto podem absorver o acréscimo da demanda sem que haja necessidade de ampliação premente de capacidade. Por outro lado, se operam com retornos crescente de escala, possíveis folgas operacionais podem ser comprometidas rapidamente, exigindo um redimensionamento da capacidade para que permaneçam sobre a fronteira da eficiência. Na prática, os aeroportos operam com retornos decrescente de escala em termos do binômio demanda X capacidade.

Quanto a possíveis folgas ou limiares de capacidade dos aeroportos posicionados sobre a fronteira da eficiência, cabe, também, considerar o perfil do aeroporto com relação à distribuição do fluxo de passageiros e aeronaves ao longo do dia. Períodos de "vale" presentes em alguns aeroportos (representam capacidade ociosa) não são captados pelo método DEA, pois a determinação das melhores relações entre capacidade e demanda é baseada somente nos valores atribuídos às variáveis de entrada e saída do modelo.

Portanto, aeroportos avaliados como eficientes dependem da magnitude do fator de escala de operação e de outros parâmetros não captados pelo modelo para avaliação da necessidade de aumento de capacidade em função de acréscimo da demanda. 
Em "Projeção de Demanda de Transporte Aéreo" (INFRAERO, 2010), são apresentadas as projeções de demanda na hora-pico para os 66 aeroportos da rede INFRAERO para 2014, 2019 e 2029, considerando três perspectivas: pessimista, média e otimista. A Tabela 10 mostra as projeções (média) de hora-pico para os dez primeiros aeroportos do ranking quanto ao movimento de passageiros, os quais representam $70,95 \%$ do total da rede.

Tabela 10 - Projeções de demanda ${ }^{18}$

\begin{tabular}{|c|c|c|c|c|}
\hline Sigla ICAO & $\begin{array}{c}\text { Hora Pico } \\
\mathbf{( 2 0 1 0 )}\end{array}$ & Hora Pico (2014) & $\begin{array}{c}\text { Hora Pico } \\
\mathbf{( 2 0 1 9 )}\end{array}$ & $\begin{array}{c}\text { Hora Pico } \\
\mathbf{( 2 0 2 9 )}\end{array}$ \\
\hline SBGR & 5503 & 6555 & 8378 & 12292 \\
\hline SBSP & 4171 & 5015 & 6338 & 9105 \\
\hline SBBR & 4046 & 4974 & 6699 & 10329 \\
\hline SBGL & 3208 & 4319 & 5497 & 8638 \\
\hline SBRJ & 2307 & 3056 & 3681 & 5545 \\
\hline SBSV & 2020 & 2308 & 3069 & 5238 \\
\hline SBCF & 2158 & 3158 & 3932 & 5860 \\
\hline SBPA & 1862 & 2337 & 3192 & 5522 \\
\hline SBRF & 1592 & 1843 & 2425 & 4000 \\
\hline SBCT & 1774 & 2128 & 2677 & 4052 \\
\hline
\end{tabular}

Observa-se que, comparativamente a 2010, a projeção para o Aeroporto de Congonhas (SBSP) é de uma taxa de crescimento médio anual da ordem de $20 \%$ em 2014 e de $118 \%$ em 2029. Para o Aeroporto Internacional Antônio Carlos Jobim (SBGL), a taxa de crescimento é de $35 \%$ e de $169 \%$ para 2014 e 2029, respectivamente. A projeção para o Aeroporto Internacional Tancredo Neves (SBCF) é ainda maior para 2014: 46\%; para 2029 o aumento é de $151 \%$.

Verifica-se, portanto, que aeroportos atualmente sobre a fronteira da eficiência e que requeiram aumento de capacidade devido ao aumento da demanda no curto ou longo prazos, podem deixar de ter as melhores relações entre produtos e insumos, quando comparados a outros pares da amostra considerada, caso não haja ações voltadas à ampliação de componentes de infraestrutura aeroportuária.

\footnotetext{
${ }^{18}$ Fonte: INFRAERO (2011d)
} 


\section{Conclusão}

O presente estudo utilizou o método Análise de Envoltória de Dados para avaliar a eficiência técnica relativa de 23 aeroportos brasileiros, tomando-se como parâmetros as relações entre a capacidade dinâmica de componentes aeroportuários e o fluxo de passageiros e aeronaves. $\mathrm{O}$ estudo apontou que 11 aeroportos operam com 100\% de eficiência quando comparados aos demais, representando 47,8\% do total da amostra. Com relação aos objetivos intermediários, o trabalho identificou os componentes processadores que comprometem o desempenho da DMU avaliada, bem como os acréscimos e reduções necessárias às variáveis do modelo, de modo a conduzir o aeroporto para a fronteira da eficiência. Verifica-se que "sala de embarque" tem o maior percentual médio em termos de necessidade de aumento de capacidade (26,32\%), enquanto que "pista de pouso e decolagem" possui o menor valor percentual médio de ineficiência $(14,47 \%)$, dentre as variáveis utilizadas no modelo.

Neste contexto, possíveis outras ações, que não exigem um redimensionamento de processadores (exclusivamente), podem corroborar com o aumento de capacidade e, consequentemente, da eficiência dos aeroportos avaliados, como a implantação de sistema de balcões de check-in compartilhados (inexistência de exclusividade de companhias aéreas na utilização de posições de balcões check-in), o aumento de totens de autoatendimento, a alocação menos concentrada dos horários (slots) de voos ao longo do dia. Porém, algumas dessas decisões extrapolam a competência do gestor do aeroporto, requerendo um entendimento conjunto entre companhias aéreas, órgãos reguladores e autoridades aeroportuárias.

As proposições para estudos futuros de desdobramento da pesquisa incluem:

- utilização de métodos paramétricos, como regressão TOBIT, tomando-se a eficiência como variável dependente e as capacidades de componentes como variáveis explanatórias, de modo a analisar a relação e a contribuição das variáveis com os níveis de eficiência (recomenda-se a ampliação da amostra);

- aplicação da técnica não paramétrica Free Disposable Hull (FDH) a fim de comparar os resultados com os da DEA; e 
- utilização da técnica DEA com todos os aeroportos da rede INFRAERO. A proposta é reunir os aeroportos em grupos, conforme o perfil de tráfego, e aplicar o modelo em cada grupo a fim de proceder-se uma análise segregada (considerar as condições da DEA quanto às relações entre o número de DMU e de variáveis).

\section{Referências}

Agência Nacional de Aviação Civil - ANAC (2007) Demanda na hora-pico - Aeroportos da Rede INFRAERO. Superintendência de Estudos, Pesquisas e Capacitação para a Aviação Civil. Rio de Janeiro. $1^{\circ}$ Edição.

Agência Nacional de Aviação Civil - ANAC (2010a) Dados Comparativos Avançados. Documento técnico não publicado. Disponível em www2.anac.gov.br.

Agência Nacional de Aviação Civil - ANAC (2009) Regulamento Brasileiro da Aviação Civil, RBAC n. 139. Brasília: ANAC. Disponível em: www.anac.gov.br.

Centro de Gerenciamento de Navegação Aérea - CGNA (2010) Capacidade de pista. Documento técnico não publicado. Disponível em www.cgna.gov.br.

Charnes, A., Cooper, W.W. e Rhodes, E. (1978) Measuring the efficiency of decision making units. European Journal of Operational Research, vol. 2, pp. 429-444.

Cooper, W., Sieford, L. e Tone, K. (2007) Data Envelopment Analysis, a comprehensive Text with Models, Applications, Reference and DEA-Solver software. New York: Editora Springer Publishers.

Doganis, R., Pearson, R. e Thompson, G. (1978) Airport Economics in the Seventies. Transport Studies Group, Research Report, n. 5. UK: Polytechnic of Central London.

Farrel, M.J. (1957) The mearurement of productive efficiency. Journal of Royal Statistical Society, vol. 120, n. 3, pp. 253-290.

Airport Development Reference Manual - IATA (2011). $9^{\circ}$ Edição.

Empresa Brasileira de Infraestrutura Aeroportuária - INFRAERO (2011a) Curso de Engenharia Aeroportuária. Documento técnico não publicado.

Empresa Brasileira de Infraestrutura Aeroportuária - INFRAERO (2011b) A INFRAERO. Disponível em: www.infraero.gov.br.

Empresa Brasileira de Infraestrutura Aeroportuária - INFRAERO (2011c) Estatística. Documento técnico não publicado. Disponivel em: www.infraero.gov.br.

Empresa Brasileira de Infraestrutura Aeroportuária - INFRAERO (2011d). Projeções de Demanda por Transporte Aéreo. Documento técnico não publicado.

Empresa Brasileira de Infraestrutura Aeroportuária - INFRAERO (2010) Metodologia para Avaliação de Capacidade de Terminais de Passageiros. Informação Padronizada n. 109-DO/1/6/2010.

Instituto de Pesquisa Econômica Aplicada - IPEA (2010) Comunicados do IPEA: Panorama e perspectivas para o transporte aéreo no Brasil e no mundo, n. 54. Brasília.

Lima de Souza A. L., Pacheco R. R. e Fernandes E. (2008) Uma análise comparativa de desempenho de aeroportos a nível mundial, SITRAER 7, pp 382-406. 
Peña, C. S. (2008) Um Modelo de Avaliação da Eficiência da Administração Pública através do Método Análise de Envoltória de Dados, Revista de Administração Contemporânea, vol. 12, n. 1, pp. 83-106.

Pires de Almeida, I. B. (2009) Análise de desempenho de escolas públicas cicladas e não cicladas pertencentes ao ensino fundamental. Tese (Doutorado), Universidade Estadual de Campinas, Campinas.

Toresan, L. (1988) Sustentabilidade e desempenho produtivo na agricultura - uma abordagem multidimensional aplicada a empresas agrícolas. Tese (Doutorado em Engenharia de Produção e Sistemas), Universidade Federal de Santa Catarina, Florianópolis.

Vicini, L. (2005) Análise multivariada da teoria à prática. Universidade Federal de Santa Maria, Santa Maria.

Wang, P.T. e Pitfield, D.E (1999) The derivation and analysis of the passenger peak hour: an empirical application to Brazil. Journal of Air Transport Management, vol. 5, pp. 135-141.

Wells A. T e Young S. B. (2004) Airport Planning and Management. New York: Editora McGrawHill. 